\title{
Humic substances narrow fractions potentiate inhibitory activity of sulbactam with respect to $\beta$-lactamase TEM-1
}

\author{
Mikhnevich T. A., ${ }^{1}$ Rubtsova M. Y., ${ }^{1}$ Grigorenko V. G., ${ }^{1}$ Rukhovich G. D., ${ }^{1}$ \\ Burykina Y. V., ${ }^{2}$ and Perminova I. V. ${ }^{1}$ \\ tanya.4erkasova8q@gmail.com \\ ${ }^{1}$ Lomonosov Moscow State University, Department of Chemistry, Moscow, Russia \\ 2Zelensky Institute of Organic Chemistry, Moscow, Russia \\ doi: 10.36291/IHSS.2021.mikhnevich
}

Keywords: SPE fractionation, FTICR MS, humic substances, beta-lactamase, inhibitor Antibiotic resistance became a global problem, while microorganisms developed several adaptive tools to escape the action of antibiotics. The most efficient tool is enzymatic degradation of $\beta$-lactam antibiotics realized via release of $\beta$-lactamases. To overcome this problem, inhibitors of $\beta$-lactamases are widely used in clinical practice. However, they are quickly losing activity for new $\beta$-lactamase mutants. Molecular ensemble of humic substances (HS) has dynamic composition which might hinder molecular recognition of $\mathrm{HS}$ as inhibitor by bacteria. The aim of this work was to isolate and characterize the most active components of HS. For achieving the goal of this study, we have synthesized and used CENTA [1] as a chromogenic substrate for studying enzymatic activity of $\beta$ lactamase TEM-1 in the presence of sulbactam, HS or mixture of them. To isolate the most active components of $\mathrm{HS}$, the hymatomelanic acids (CHM) were isolated from commercial sample of coal potassium/sodium humate (Humate-Extra) using ethanol extraction. The narrow fractions of $\mathrm{CHM}$ were obtained by polarity driven separation with a use of solid-phase extraction and gradient elution with water-methanol eluate with a stepwise increase in the amount of methanol from 30 to $100 \%$. Fourier transform ion cyclotron resonance mass spectrometry (FTICR MS) performed using FTICR mass spectrometer $15 \mathrm{~T}$ solariX (Bruker Daltonics) for all samples. Molecular assignments were made using lab-made Transhumus software and plotted into van Krevelen diagrams. The samples were also characterized by fluorescence spectroscopy.

No significant inhibitory effect was found for $\mathrm{CHM}$ and its narrow fractions in this study, however, a regular synergistic increase in inhibition was observed in the presence of sulbactam (Fig. 1.)

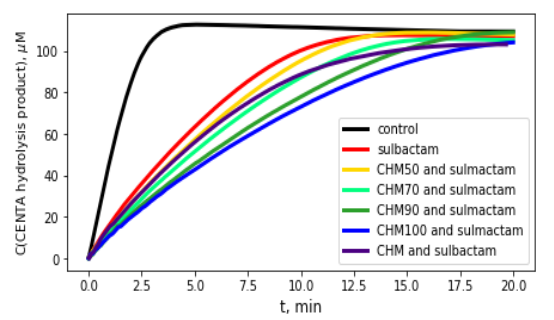

Fig. 1. Kinetic curves of CENTA $(100 \mu M)$ hydrolysis by $\beta$ lactamase TEM-1 $7 \mathrm{nM}$ ): in the absence of inhibitors (control, highlighted in black) and in the presence of sulbactam ( $5 \mu \mathrm{M}$, red), CHM and its narrow fractions (50 mg.L-1, purple, yellow, light green, green and blue). Some data are omitted to avoid overlap of the curves.

Molecular compositions of the fractions varied substantially in chemical diversity. The most apolar fractions are characterized by a higher content of aromatic and lowoxidized compounds. Also, with an increase in the non-polarity of the fractions, a decrease in the fluorescence emission band asymmetry $\left(\lambda_{\mathrm{ex}}=280 \mathrm{~nm}\right)$ was observed, which may indicate a substantial decrease in the degree of humification and condensation of HS molecules.

Thus, an increase in the contribution of apolar (hydrophobic and amphipatic) components in the humic ensemble led to an increase in the synergistic effect with respect to sulbactam. Revealing mechanism of enhancing inhibitory activity of sulbactam in the presence of HS is a subject of our future studies.

\section{Acknowledgements}

This work was supported by grant from the Russian Science Foundation № 21-73-20202. FTICR MS measurements were carried out using the equipment of the Shared Use Center of the Institute of Organic Chemistry of the Russian Academy of Sciences.

[1] Bebrone C. et al. Antimicrob. Agents Chemother. 2001, 45 (6), 1868-1871. 